\title{
ON RESULTANTS
}

\author{
GERALD MYERSON
}

\begin{abstract}
Let $f$ and $g$ be polynomials with coefficients in a commutative ring $A$. Let $f$ be monic. We show that the resultant of $f$ and $g$ equals the norm from $A[x] /(f)$ to $A$ of $\bar{g}$. As a corollary we deduce that if $c$ is in $A$ and also in the ideal generated by $f$ and $g$, then the resultant divides $c^{n}$, where $n$ is the degree of $f$.
\end{abstract}

In this paper $A$ will denote a commutative ring with unity. Let $f$ be a monic polynomial with coefficients in $A$. Let $B=A[x] /(f)$. Given $\alpha$ in $B$ the norm of $\alpha$, denoted $N(\alpha)$, is defined to be the determinant of the right regular representation of $\alpha$.

Now let $f(x)=\sum_{j=0}^{n} a_{n-j} x^{j}$ and $g(x)=\sum_{j=0}^{m} b_{m-j} x^{j}$ be polynomials with coefficients in $A$. The resultant of $f$ and $g$, denoted $R(f, g)$, is given by:

$$
R(f, g)=\operatorname{det}\left(\begin{array}{cccccccc}
a_{0} & a_{1} & \cdot & \cdots & a_{n} & & & \\
& a_{0} & & \cdots & \cdot & a_{n} & & \mathbf{0} \\
& & \ddots & & & & \ddots & \\
\mathbf{0} & & & a_{0} & \cdots & \cdot & & a_{n} \\
b_{0} & b_{1} & \cdot & \cdots & b_{m} & & & \\
& b_{0} & & \cdots & \cdot & b_{m} & & \mathbf{0} \\
& & \ddots & & & & \ddots & \\
& 0 & & b_{0} & \cdot & \cdots & & b_{m}
\end{array}\right) \text { rows }
$$

THEOREM. Let $f$ and $g$ be polynomials with coefficients in $A, f$ monic. Let $\bar{g}$ be the class of $g$ in $A[x] /(f)$. Then $N(\bar{g})=R(f, g)$.

Proof. On the hypotheses, the first $m$ terms on the diagonal of the matrix above are ones. Gaussian elimination applied to the matrix, using these elements as pivots, yields $R(f, g)=\operatorname{det}\left(\begin{array}{l}I \\ 0 \\ 0\end{array}\right)$, where $I$ is the $m \times m$ identity matrix. Thus, $R(f, g)=$ det $M$. But it is easily checked that $M$ is the transpose of the right regular representation of $\bar{g}$.

COROLlaRY. If $f, g, r$ and $s$ are polynomials with coefficients in $A$, and $f$ is monic then $R(f, f r+g s)=R(f, g) R(f, s)$.

Received by the editors October 26, 1982 and, in revised form, January 12, 1983.

1980 Mathematics Subject Classification. Primary 13B25, $10 \mathrm{M} 05$.

Key words and phrases. Resultants.

(C)1983 American Mathematical Society $0002-9939 / 83 \$ 1.00+\$ .25$ per page 
Remark. This is well known, at least when $A$ is taken to be a field. The theorem above permits a one-line proof.

Proof. $R(f, f r+g s)=N(\overline{f r+g s})=N(\overline{g s})=N(\bar{g}) N(\bar{s})=R(f, g) R(f, s)$.

COROLlary. Let $f$ and $g$ be as in the Theorem above. Let $J=J(f, g)$ be the ideal generated by $f$ and $g$ in $A[x]$, and let $c$ be an element of $J \cap A$. Then $R(f, g)$ divides $c^{n}$, where $n$ is the degree of $f$.

Remark. That $R(f, g)$ divides some power of $c$ is known (see e.g. [2, Lemma 11.3]). In transcendence theory it is often helpful to have bounds for a nonzero resultant (see, e.g. [1]). We present the Corollary in the spirit of such bounds.

Proof. Let $f, g$ and $c$ be as given. Then there exist polynomials $r$ and $s$ with coefficients in $A$ such that $c=f r+g s$. Then $c^{n}=R(f, c)=R(f, f r+g s)=$ $R(f, g) R(f, s)$, whence $R(f, g)$ divides $c^{n}$.

This Corollary is sharp, in the following sense. Given any nonunit $c$ in $A$, and any positive integer $n$, there exist $f$ and $g$ in $A[x], f$ monic of degree $n$, such that $c$ is in $J(f, g)$, and $R(f, g)$ does not divide $c^{n-1}$. For example, take $f(x)=x^{n}, g(x)=x^{n}$ $+c$; then $R(f, g)=c^{n}$.

Moreover, it is not possible, in general, to remove the condition that $f$ be monic. Take for $A$ the integers, let $f(x)=2 x+1$, and let $g(x)=2 x+17$. Then 1 is in $J(f, g)$, since

$$
x^{4} g(x)-\left(x^{4}+8 x^{3}-4 x^{2}+2 x-1\right) f(x)=1 .
$$

However, $R(f, g)=32$. The construction exemplified here is clearly quite general.

If it is required that the leading coefficients of $f$ and $g$ be relatively prime, it can be shown that if $c$ is in $A \cap J(f, g)$ then $R(f, g)$ divides $c^{k}$, where $k=\max (m, n)$.

We note as a further corollary that, under the assumption that $f$ is monic, $J(f, g)$ contains $A$ if and only if $R(f, g)$ is a unit. We would like to propose the problem of characterizing those pairs $f, g$ for which $R(f, g)$ is a unit or, more generally, for which $R(f, g)$ divides every element of $A \cap J(f, g)$.

We would like to acknowledge the helpful comments of the referee and editor, which led us to an extensive revision of our original manuscript.

\section{REFERENCES}

1. W. D. Brownawell, Some remarks on semi-resultants, Transcendence Theory: Advances and Applications (A. Baker and D. W. Masser, eds.), Academic Press, New York, 1977. MR 58 \# 537.

2. L. N. Vaseršteĭn and A. A. Suslin, Serre's problem on projective modules over polynomial rings, and algebraic K-theory, Math. USSR-Izv. 10 (1976), 937-1001. MR 56 \#560.

Department of Mathematics, University of British Columbia, Vancouver, British Columbia, CANADA V6T 1Y4

Current address: Department of Mathematics, State University of New York at Buffalo, Buffalo, New York 14214-3093 